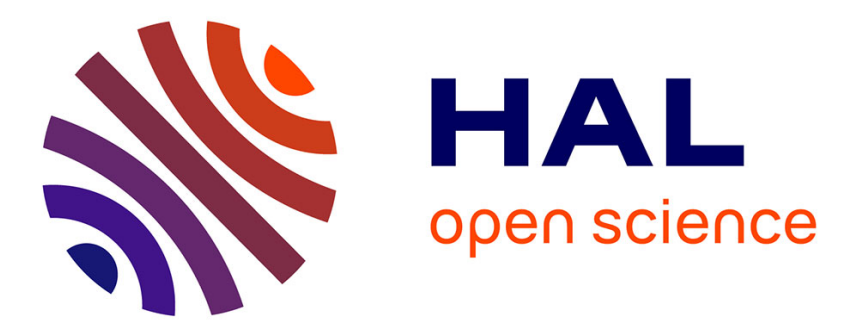

\title{
Comportement de quatre variétés de blé tendre vis-à-vis de la fusariose de l'épi causée par Fusarium roseum var. culmorum (Schwabe) Sn. et $\mathbf{H}$
}

\author{
Laurent Saur, Jean-Yves Morlais
}

\section{- To cite this version:}

Laurent Saur, Jean-Yves Morlais. Comportement de quatre variétés de blé tendre vis-à-vis de la fusariose de l'épi causée par Fusarium roseum var. culmorum (Schwabe) Sn. et H. Agronomie, 1984, 4 (10), pp.939-943. hal-00884597

\section{HAL Id: hal-00884597 \\ https://hal.science/hal-00884597}

Submitted on 1 Jan 1984

HAL is a multi-disciplinary open access archive for the deposit and dissemination of scientific research documents, whether they are published or not. The documents may come from teaching and research institutions in France or abroad, or from public or private research centers.
L'archive ouverte pluridisciplinaire HAL, est destinée au dépôt et à la diffusion de documents scientifiques de niveau recherche, publiés ou non, émanant des établissements d'enseignement et de recherche français ou étrangers, des laboratoires publics ou privés. 


\title{
Comportement de quatre variétés de blé tendre vis-à-vis de la fusariose de l'épi causée par Fusa- rium roseum var. culmorum (Schwabe) Sn. et $\mathrm{H}$.
}

\author{
Laurent SAUR \\ avec la collaboration technique de Jean-Yves MorLaIs
}

I.N.R.A., Station d'Amélioration des Plantes, Centre de Recherches de Rennes, B.P. 29, F 35650 Le Rheu

\begin{abstract}
Quatre variétés de blé tendre d'hiver de sensibilité différente vis-à-vis de la fusariose de l'épi ont été soumises à 3 types d'infection artificielle : par pulvérisation, directement dans un épillet soit du sommet, soit de la base de l'épi.

L'infection par pulvérisation montre une différence entre les variétés pour la résistance à la pénétration et la résistance à l'extension des symptômes à partir d'un point de pénétration (tabl. 2). L'infection au sommet n'a pas d'action dépressive sur la partie saine de l'épi (tabl. 3). L'infection à la base diminue le poids de 1000 grains de la partie saine pour la seule variété " Réso ", ce qui correspond à une plus grande sensibilité à l'envahissement du rachis. La variété "Bizel » présente un bon niveau de résistance à la pénétration et à l'extension des symptômes.
\end{abstract}

Mots clés additionnels : Fusarium culmorum Link, résistance, Triticum aestivum.

\begin{abstract}
Behaviour of four wheat cultivars towards head blight caused by Fusarium roseum var. culmorum (Schwabe) Sn. et $H$.

Four winter wheat cultivars with different levels of susceptibility to fusarium head blight were inoculated in three ways : inoculation by spraying, inoculation of a single spikelet in the upper, or lower, part of the ear. Inoculation by spraying gave differences in varietal reactions for resistance to initial infection and resistance to the spread of the infection within the ear (table 2). Inoculation of the top of the ear had no incidence on thousand grain weight of the undamaged part of the ear (table 3). Inoculation of the lower part of the ear decreased thousand grain weight of the undamaged part of the ear only for cv "Réso" (table 4). This means that this cultivar is very susceptible to colonization of the rachis by the fungus. Cultivar "Bizel" has good resistance to both types : initial infection and spread of the infection.
\end{abstract}

Additional key words : Fusarium culmorum Link, resistance, scab, Triticum aestivum.

\section{INTRODUCTION}

La fusariose de l'épi provoquée par Fusarium roseum (Link) Sn. et $\mathrm{H}$. se manifeste chez le blé par le dessèchement précoce de certains épillets. On distingue plusieurs variétés au sein de l'espèce $F$. roseum. Les deux principales parasitant les épis de blé sont $F$. roseum var. culmorum (Schwabe) Sn. et $\mathrm{H}$. et $F$. roseum var. graminearum (Schwabe) Sn. et H. (MESSIAEN \& CASSINI, 1968). Ces 2 variétés diffèrent par leur biologie mais donnent des symptômes identiques. On n'a pas signalé jusqu'à présent de variétés de blé présentant un comportement différent vis-à-vis de ces 2 parasites. $F$. roseum var. graminearum est prépon- dérant dans la moitié Sud de la France, $F$. roseum var. culmorum dans la moitié Nord. Le parasite contamine les épis par voie aérienne. Le développement de la maladie dépend du stade de la plante et de l'existence d'une humidité élevée au moment de la contamination. Le stade de sensibilité maximum se situe à l'anthèse (ANDERSEN, 1948; LEIJERSTAM, 1962 ; SAUR \& TROTTET, 1981).

On ne connaît pas de haut niveau de résistance au sein de l'espèce blé tendre mais des différences entre variétés ont été mises en évidence (DICKSON, 1942 ; SCHROEDER \& CHRISTENSEN, 1963 ; BOCKMANN et $a l .$, 1975). SCHROEDER \& CHRISTENSEN (1963) ont montré, sur 7 lignées de blé de printemps, que le com- 
portement vis-à-vis de la fusariose de l'épi correspondait à 2 phénomènes : résistance à la contamination et résistance à l'extension de la maladie.

Le but de cette étude est de préciser le comportement de 4 variétés de blé tendre d'hiver vis-à-vis d'une attaque sur épi de $F$. roseum var. culmorum. Ces variétés ont été choisies, après études préalables, pour représenter la gamme de réaction d'une collection de variétés d'origine française.

\section{MATÉRIEL ET MÉTHODES}

Deux essais ont été réalisés au champ en 1978 (essai $n^{\circ} 1$ ) et 1979 (essai $n^{\circ} 2$ ). Les 4 variétés étudiées sont " Bizel », " Capest ", " Top » et « Réso » représentant 2 groupes de précocité (tabl. 1).
Pour les 2 essais, le dispositif expérimental est un criss-cross à 4 répétitions. Les parcelles élémentaires sont constituées de 3 lignes de $1,5 \mathrm{~m}$ à $20 \mathrm{~cm}$ d'intervalle et séparées par 2 lignes de bordure. Pour chaque répétition, un des sous-blocs est infecté et l'autre sert de témoin.

L'inoculum est constitué d'une suspension de spores obtenue à partir d'une culture pure d'une souche du parasite sur grains d'orge humides stérilisés selon la technique de BOCKMANN (1962). Les spores sont récupérées par lavage et la concentration ajustée à $10^{6}$ spores $/ \mathrm{ml}$. La contamination est réalisée à l'anthèse pour chaque variété. Des apports de $2 \mathrm{~mm}$ d'eau sont effectués avant l'infection à l'aide d'un dispositif d'irrigation par aspersion puis répétés toutes les $48 \mathrm{~h}$

TABLEAU

Origine génétique et précocité des variétés étudiées.

Genetic origin and earliness of the cultivars.

\begin{tabular}{|c|c|c|c|c|}
\hline \multirow{2}{*}{ Variétés } & \multirow{2}{*}{$\begin{array}{c}\text { Année } \\
\text { d'inscription }\end{array}$} & \multirow{2}{*}{ Origine génétique } & \multicolumn{2}{|c|}{ Date d'épiaison } \\
\hline & & & 1978 & 1979 \\
\hline Bizel & 1966 & (Blé bohémien $\times$ Blé seigle $) \times$ Oro $\times$ Variant de Hauters & $4 / 6$ & $7 / 6$ \\
\hline Capest & 1967 & $(90 \times$ Etoile de Choisy) $\times(2-7 \times$ Cappelle $)$ & $4 / 6$ & $7 / 6$ \\
\hline Top & 1970 & (Providence $\times$ Vilmorin 27$) \times($ Etoile de Choisy $\times$ Cappelle $)$ & $27 / 5$ & $30 / 5$ \\
\hline Réso & 1974 & FD $66 \times$ Heine 2806 & $28 / 5$ & $1 / 6$ \\
\hline
\end{tabular}

L'essai $n^{\circ} 1$ est infecté par pulvérisation jusqu'au début de ruissellement, ce qui correspond à la dose approximative de $100 \mathrm{ml} / \mathrm{m}^{2}$. Pour chaque parcelle, 50 épis à l'anthèse sont marqués le jour de l'infection. Sur les parcelles infectées, les épis sont repérés individuellement par un numéro et 9 notations sont effectuées entre l'infection et le début de la maturité. Le nombre de points de pénétration est estimé comme suit :

- Lors de la $1^{\text {re }}$ notation, les épillets présentant une nécrose caractéristique d'un début de symptôme sont dénombrés.

- Lors des notations suivantes, certains épillets sont déjà complètement desséchés. L'extension des symptômes se manifeste par un dessèchement des épillets contigus ou opposés. Sont comptés comme points de pénétration, en plus des épillets présentant une nécrose, les épillets desséchés qui ne sont ni contigus ni opposés à un épillet précédemment fusarié.

A maturité, les épis marqués sont récoltés et pesés. Le poids de 50 épis de la parcelle infectée est exprimée en p. 100 de celui de la parcelle témoin.

L'essai $n^{\circ} 2$ est infecté en déposant à l'aide d'une seringue une goutte d'inoculum dans la $1^{\text {re }}$ fleur d'un épillet selon 2 modalités :

- dans l'épillet subterminal de l'épi,

- dans un épillet de la base,

50 épis par parcelle ont été infectés à l'anthèse et, le même jour, 50 épis à l'anthèse sont marqués dans les parcelles témoin. Le nombre d'épillets fusariés est noté avant maturité. Après récolte, les épis sont pesés puis, pour chaque épi, on supprime les épillets présentant des symptômes en éliminant chaque fois les épillets correspondants sur un épi de la parcelle témoin. Pour les 50 épis issus d'une parcelle élémentaire, les parties saines sont battues en mélange et on mesure le poids de 1000 grains.

Les résultats ont été analysés selon la technique de l'analyse de variance.

\section{RÉSULTATS}

Les attaques naturelles de $F$. roseum ont été très faibles dans les parcelles témoin. L'irrigation par aspersion a permis un très bon développement de la fusariose de l'épi dans les parcelles infectées, surtout en 1978 .

\section{A. Infection par pulvérisation}

Les résultats des dernières notations avant maturité ainsi que l'influence de l'infection sur le poids des épis sont donnés dans le tableau 2. Le nombre d'épillets fusariés permet de classer nettement les 4 variétés étudiées. "Bizel » s'avère peu sensible et "Réso » très sensible. Le poids d'épi est très affecté pour toutes les variétés, ce qui permet de les classer en utilisant le rapport infecté/témoin. L'écart entre la plus sensible et la moins sensible est très important; le classement des variétés est le même dans les 2 cas. Le nombre de points de pénétration ne permet pas de distinguer " Capest ", " Top » et " Réso » mais est nettement inférieur pour « Bizel ». L'étude du rapport du nombre d'épillets fusariés au nombre de points de pénétration qui représente l'extension des symptômes met en évidence une différence entre «Bizel» et les autres variétés, ainsi qu'entre «Top» et «Réso». Les variétés étudiées présentent donc un comportement assez différent :

- «Bizel » diffère des 3 autres variétés pour sa 
TABLEAU 2

Infection par pulvérisation: nombre d'épillets fusariés, nombre de points de pénétration, poids de 50 épis en p. 100 du témoin.

Inoculation by spraying : number of damaged spikelets, number of spots of initial infection, 50 ear weight per cent of the control.

\begin{tabular}{|c|c|c|c|c|}
\hline Variétés & $\begin{array}{l}\text { Nombre d'épillets } \\
\text { fusariés }\end{array}$ & $\begin{array}{l}\text { Nombre de points } \\
\text { de pénétration }\end{array}$ & $\begin{array}{l}\text { Nombre d'épillets } \\
\text { fusariés/Nombre de } \\
\text { points de pénétration }\end{array}$ & $\begin{array}{l}\text { Poids de } 50 \text { épis } \\
\text { infestés/témoin }(\%)\end{array}$ \\
\hline Bizel & $94\}(1)$ & $52\}$ & $1,45\}$ & $93,3\}$ \\
\hline Top & $593\}$ & 168) & $3,56\}$ & $71,3\}$ \\
\hline Capest & $719\}$ & $172\}$ & $4,23\}\}$ & $57,3\}$ \\
\hline Réso & $890\}$ & $180\}$ & $4,98\}$ & $36,9\}$ \\
\hline \multirow{2}{*}{$\begin{array}{l}\text { Analyse de variance } F \\
\text { Coefficient moyen } \\
\text { de variation }\end{array}$} & $164,1 \mathrm{~S}(2)$ & $68,7 \mathrm{~S}$ & $41,9 \mathrm{~S}$ & $63,2 \mathrm{~S}$ \\
\hline & 9,3 & 10,3 & 13,2 & 9,2 \\
\hline
\end{tabular}

(1) Les variétés reliées par un même trait ne diffèrent pas significativement au seuil 5 p. 100.

(2) $\mathrm{S}=$ significatif au seuil 5 p. 100.

résistance à la pénétration et sa résistance à l'extension des symptômes,

— « Top » diffère de " Réso » pour la résistance à l'extension des symptômes.

La figure 1 présente le nombre total d'épillets fusariés pour 50 épis aux différentes dates de notation. Huit jours après l'infection, « Capest », « Top » et «Réso » présentent des symptômes nets alors que les premières traces n'apparaissent sur « Bizel » qu'au bout de 11 jours. Les courbes d'évolution sont parallèles pour "Capest" et "Réso ». La différence observée à la dernière notation correspond à la différence de date d'infection. A partir du 11 juillet, la notation devient impossible sur "Top » et " Réso » et difficile sur «Bizel » et « Capest » en raison de l'état de maturité.

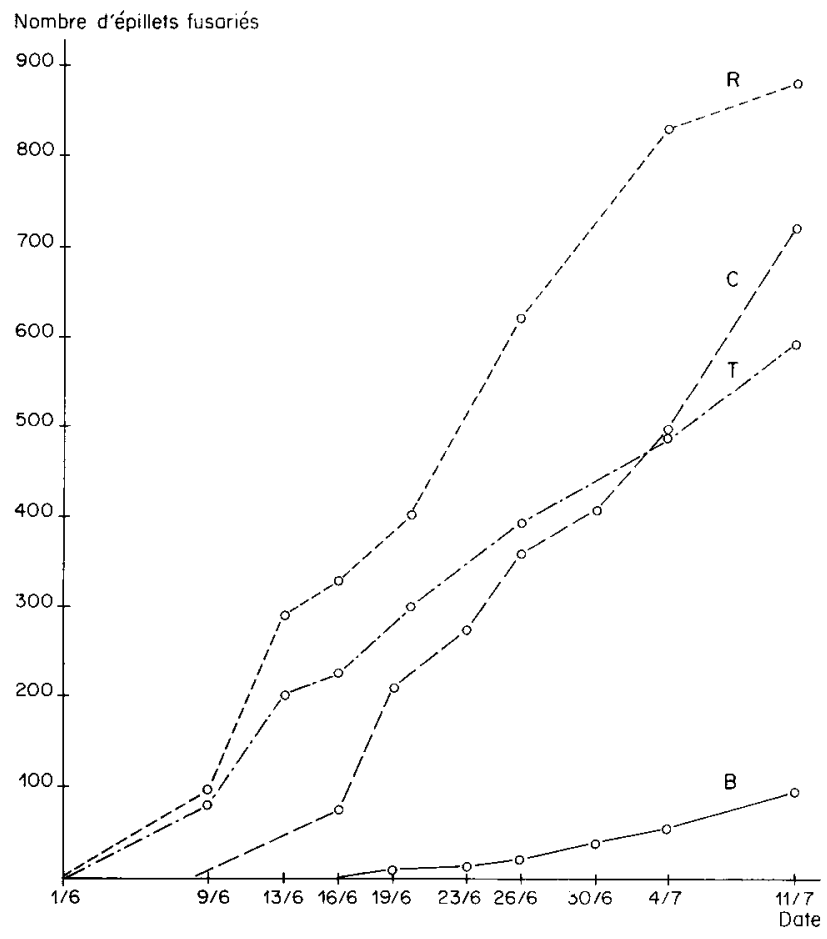

Figure 1

Nombre d'épillets fusariés aux différentes dates de notation pour 50 épis de "Bizel " (B), " Capest " (C), "Réso " (R), "Top » (T). Number of damaged spikelets in 50 ears as affected by time after inoculation.

\section{B. Infection localisée}

\section{Infection dans un épillet subterminal (tabl. 3)}

L'extension des symptômes est faible à partir de l'épillet infecté, sauf pour la variété "Réso ». De même, seule cette variété présente une diminution significative de poids d'épi dans la parcelle infectée. Le poids de 1000 grains de la partie saine de l'épi est légèrement influencé par l'infection dans le sens d'une augmentation ; aucune interaction variété $\times$ infection n'est mise en évidence.

\section{Infection dans un épillet de la base (tabl. 4)}

L'extension des symptômes est faible sauf pour "Réso ». L'infection diminue le poids de 50 épis en particulier pour " Capest " et "Réso " mais aucune interaction variété $x$ infection n'est mise en évidence. L'essai révèle une action de l'infection sur le poids de 1000 grains de la partie saine de l'épi pour la seule variété "Réso ». Pour cette variété, l'effet sur le poids de l'épi s'explique par le nombre d'épillets fusariés et par l'échaudage des grains de la partie supérieure de l'épi.

\section{DISCUSSION}

La dernière notation de symptômes a été réalisée à la même date pour les 4 variétés. Il aurait été souhaitable de faire une notation supplémentaire pour les variétés tardives ("Bizel » et "Capest ») correspondant au même nombre de jours après l'infection que pour les variétés précoces ( Top » et « Réso »). Cette dernière notation s'est avérée impossible en raison de l'état de maturité de l'épi. En 1978, la durée de la période de floraison-maturité a été inférieure pour « Bizel » et « Capest ». Le moindre développement de la maladie sur "Capest " par rapport à "Réso ", auquel correspond une moindre chute de rendement, pourrait s'expliquer par une différence dans la durée de la phase floraison-maturité.

En infection localisée, l'extension des symptômes est faible et plus importante pour " Réso » que pour «Top » et " Capest ». Le faible nombre d'épillets fusariés refléterait le fait que la goutte d'inoculum déposée dans la fleur peut détruire le grain sans qu'il $\mathrm{y}$ ait réelle pénétration du parasite. La variété 
TABLEAU 3

Infection au sommet de l'épi : nombre d'épillets fusariés, poids de 50 épis, poids de I 000 grains de la partie saine de l'épi. Inoculation into a spikelet of the top of the ear : number of damaged spikelets, 50 ear weight, thousand grain weight of the undamaged part of the ear.

\begin{tabular}{|c|c|c|c|c|c|}
\hline \multirow[t]{2}{*}{ Variétés } & \multirow{2}{*}{$\begin{array}{c}\text { Nombre d'épillets } \\
\text { fusariés (pour } 50 \text { épis) }\end{array}$} & \multicolumn{2}{|c|}{ Poids de 50 épis } & \multicolumn{2}{|c|}{$\begin{array}{l}\text { Poids de } 1000 \text { grains } \\
\text { de la partie saine de l'épi }\end{array}$} \\
\hline & & Inoculé & Témoin & Inoculé & Témoin \\
\hline Bizel & 65 & 95,2 & 91,0 & 46,3 & 44,5 \\
\hline Top & 140 & 87,9 & 89,0 & 55,3 & 53,2 \\
\hline Capest & 115 & 101,9 & 99,5 & 50,5 & 48,0 \\
\hline Réso & 375 & 97,4 & $109,6(1)$ & 51,9 & 52,4 \\
\hline \multicolumn{6}{|l|}{ Analyse de variance } \\
\hline \multirow{4}{*}{$\begin{array}{l}\mathrm{F} \text { traitement } \\
\mathrm{F} \text { variété } \\
\mathrm{F} \text { interaction } \\
\text { Coefficient moyen } \\
\text { de variation }\end{array}$} & \multirow{3}{*}{$14,6 \mathrm{~S}$} & \multicolumn{2}{|l|}{$0,5 \mathrm{NS}(2)$} & \multicolumn{2}{|l|}{$46,6 \mathrm{~S}$} \\
\hline & & \multicolumn{2}{|l|}{$13,1 \mathrm{~S}$} & \multicolumn{2}{|l|}{$47,1 \mathrm{~S}$} \\
\hline & & \multicolumn{2}{|l|}{$3,9 \mathrm{~S}$} & \multicolumn{2}{|l|}{1,5} \\
\hline & 46,1 & \multicolumn{2}{|l|}{5,5} & \multicolumn{2}{|l|}{3,1} \\
\hline
\end{tabular}

(1) Différence inoculé-témoin significative au seuil 5 p. 100.

(2) $\mathrm{S}=$ significatif au seuil 5 p. 100 . NS $=$ non significatif.

\section{TABLEAU 4}

Infection à la base de l'épi : nombre d'épillets, poids total et poids de 1000 grains de la partie saine de l'épi. Inoculation into a spikelet of the lower part of the ear : number of damaged spikelets, 50 ear weight and thousand grain weight of the undamaged part of the spike.

\begin{tabular}{|c|c|c|c|c|c|}
\hline \multirow[t]{2}{*}{ Variétés } & \multirow{2}{*}{$\begin{array}{c}\text { Nombre d'épillets } \\
\text { fusariés (pour } 50 \text { épis) }\end{array}$} & \multicolumn{2}{|c|}{ Poids de 50 épis } & \multicolumn{2}{|c|}{$\begin{array}{l}\text { Poids de } 1000 \text { grains } \\
\text { de la partie saine de l'épi }\end{array}$} \\
\hline & & Inoculé & Témoin & Inoculé & Témoin \\
\hline Bizel & 50 & 86,5 & 91,6 & 42,4 & 43,7 \\
\hline Top & 65 & 85,5 & 89,9 & 52,5 & 51,7 \\
\hline Capest & 115 & 91,6 & 103,5 & 46,3 & 48,2 \\
\hline Réso & 225 & 95,4 & 109,4 & 46,7 & $49,2(1)$ \\
\hline \multicolumn{6}{|l|}{ Analyse de variance } \\
\hline \multirow{4}{*}{$\begin{array}{l}F \text { traitement } \\
F \text { variété } \\
F \text { interaction } \\
\text { Coefficient moyen } \\
\text { de variation }\end{array}$} & & $27,4 \mathrm{~S}(2)$ & & $5,4 \mathrm{NS}$ & \\
\hline & $6,6 \mathrm{~S}$ & $23,4 \mathrm{~S}$ & & $89,5 \mathrm{~S}$ & \\
\hline & & $2,7 \mathrm{NS}$ & & $3,5 \mathrm{~S}$ & \\
\hline & 55,6 & 4,4 & & 2,4 & \\
\hline
\end{tabular}

(1) Différence inoculé-témoin significative au seuil 5 p. 100 .

(2) $\mathrm{S}=$ significatif au seuil 5 p. 100 . NS = non significatif.

« Réso », au contraire, serait plus sensible à ce type d'infection.

Pour la variété «Réso », l'effet dépressif sur le poids de 1000 grains de la partie saine de l'épi dans le cas d'une infection à la base et l'absence d'effet dans le cas d'une infection au sommet laisse penser que la seule action indirecte du parasite est un blocage de l'alimentation de la partie supérieure de l'épi dû probablement à l'envahissement du rachis. On observe même pour l'ensemble des 4 variétés une action positive sur le reste de l'épi pour une infection au sommet, ce qui peut s'interpréter comme un phénomène de compensation.

\section{CONCLUSION}

Le comportement vis-à-vis d'une attaque de fusariose de l'épi fait intervenir au moins 3 phénomènes:

- la résistance à la pénétration,
- la résistance à l'extension des symptômes,

- la résistance à l'échaudage de la partie de l'épi située au-dessus d'un épillet attaqué.

L'étude de 4 variétés de blé tendre d'hiver de sensibilité très différente a montré qu'une variabilité intergénotype existe pour ces 3 types de résistance.

La variété « Bizel » présente l'intérêt d'allier résistance à l'extension des symptômes et résistance à la pénétration. On observe de plus sur cette variété que le temps entre l'infection et l'apparition des premiers symptômes est supérieur à celui de la variété sensible " Capest ». « Bizel» est un peu tardif par rapport aux variétés françaises. Il doit être possible par sélection d'obtenir des génotypes plus précoces alliant la résistance à l'extension des symptômes mais surtout la résistance à la pénétration de « Bizel». Des croisements dans ce sens sont en cours de sélection. 


\section{RÉFÉRENCES BIBLIOGRAPHIQUES}

Andersen A. L., 1948. The development of Gibberella zeae headblight of wheat. Phytopathology, 38, 595-611.

Bockmann M., 1962. Künstliche Freilandinfektionen mit den Erregern der Fuss- und Ährenkrankheiten des Weizens. I. Vorbereitung und Durchführung der Feldinfektionen sowie deren Neben- und Nachwirkungen. Nachrichten Dtsch. Pflanzenschutz, 14, 153-156.

Bockmann M., Mielke H., Wachholz G., 1975. Untersuchungen über die Anfälligkeit verschiedener Winter- und Sommerweizensorten gegen Septoria nodorum Berk. und Fusarium culmorum Link. Zeit. Pflanzenzüchtg, 74, 39-47.

Dickson J. G., 1942. Scab of wheat and barley and its control. USDA Dept. Agr. Farmers' Bull. n ${ }^{\circ}$ 1599, 1-17.
Leijerstam B., 1962. Studies on a method of testing the resistance of wheat varieties to fusarial head blight. Wäxtskyddsanstalt. Medd., 12, 90, 181-203.

Messiaen C. M., Cassini R., 1968. Recherches sur les fusarioses. IV. La systématique des Fusarium. Ann. Epiphyt., 19, 387-384.

Saur L., Trottet M., 1981. Problèmes posés par le jugement du comportement de lignées de blé tendre et espèces voisines vis-à-vis de deux parasites de l'épi Septoria nodorum Berk. et Fusarium roseum (Link.) Sn. et $\mathrm{H}$. Mise en évidence de quelques sources de résistance. C.R. Acad. Agric. Fr., 67, 139-145.

Schroeder H. W., Christensen J. J., 1963. Factors affecting resistance of wheat to scab caused by Gibberella zeae (Schw.) Petch. Phytopathology, 53, 831-838. 